\title{
High speed confined granular flows down inclined: numerical simulations
}

\author{
Velotiana Jean-Luc RALAIARISOA ${ }^{1}$, Alexandre VALANCE ${ }^{1}$, Nicolas BRODU ${ }^{2}$, and Renaud DELANNAY ${ }^{1, \star}$ \\ ${ }^{1}$ Institut de Physique de Rennes, Université de Rennes 1, UMR CNRS 6251, 35042 Rennes Cedex, France \\ ${ }^{2}$ GEOSTAT, Inria Bordeaux, 33405 Talence, France
}

\begin{abstract}
New regimes in high-speed confined granular flows down inclined have recently been obtained in numerical simulations [1]. Increasing the angle of inclination reveals the destabilization of the well known unidirectional flows. Longitudinal rolls first appear. Upon further increase of the angle, a new regime, called supported, is observed. It is characterized by a dense core surrounded by granular gas. These numerical simulations have been performed for a fixed confinement width, $W=68 D$, where $D$ is the size of the grains. Here, we perform numerical simulations with a smaller value of the confinement width: $W=34 \mathrm{D}$. In spite of this strong confinement, we observe the transitions to the same regimes (rolls and then supported) by increasing the inclination angle. We characterize these transitions and highlight the robustness of the mass flow rate scaling law discovered in [1].
\end{abstract}

\section{Introduction}

Recently, several works have been devoted to the effect of lateral confinement on the properties of granular flows [24]. Both experimental and numerical studies have pointed out that frictional lateral walls induce new flow properties. For example, steady and fully developed flows (SFD flows) have been observed up to large angles of inclination whereas accelerated ones are usually expected $[1,5]$. These SFD regimes present non-trivial features, including secondary flows (rolls) and flows with dense core supported by a very agitated dilute layer. Despite the diversity of the features of these states the velocity of the flow obeys a simple scaling law with the flow height. In [1] the effect of the variation of the inclination angle and of the mass holdup was explored extensively and systematically for a given value of the gap $W$ between side walls. The domains of existence of the different regimes were reported for this value of $W$. We investigate here a smaller value $W / 2$ of the gap to study the effect of a variation of the gap on the domains of existence of the flows regime. We also examine if the velocity scaling law observed in [1] remains valid for this smaller value of the gap.

\section{Numerical method and simulation setup}

We have conducted $[1,5]$ simulations of granular flows down flat and steep inclines with frictional sidewalls using a discrete element model (DEM). The principle of DEM simulations is to treat each grain as a sphere (of diameter $D$ ) subject to gravity and contact forces with both the other grains and the basal and lateral walls. Interaction model is performed by using linear visco-elastic ap-

\footnotetext{
^e-mail: renaud.delannay @univ-rennes1.fr
}

proach. Two grains $i$ and $j$ interact when overlapping, such as $\delta_{i j}=\left[\left(D_{i}+D_{j}\right) / 2\right]-r_{i j}>0$, with $r_{i j}$ the centerto-center grains separation. The force applied by grain $i$ on grain $j$ is decomposed into normal $\mathbf{F}_{n}^{i \rightarrow j}$ and tangential $\mathbf{F}_{t}^{i \rightarrow j}$ components. The normal contact force is given by: $F_{n}^{i \rightarrow j}=\left(k_{n} \delta_{i j}+\gamma_{n} v_{n}\right)$ where $k_{n}$ is a spring constant, $\gamma_{n}$ a damping coefficient set by the normal coefficient of restitution $e_{n}$, and $v_{n}$ the normal component of the relative translational grain velocity. Similar model is used for the tangential component enforced by the Coulomb friction $\left|\mathbf{F}_{t}\right| \leq \mu\left|\mathbf{F}_{n}\right|$ where $\mu$ is the model friction coefficient. The torque acting on a grain is given by: $\mathbf{q}=-(D / 2)\left(\mathbf{F}_{t} \times \mathbf{n}\right)$, where $\mathbf{n}$ is the normal unit vector. Table 1 shows parameters values used for the contact forces between interacted grains and for grain-wall interactions.

Table 1: Simulation parameters used for grain/grain and grain (glass)/wall interaction model.

\begin{tabular}{lll}
\hline & Grain/grain & Grain/wall \\
\hline Normal restitution $e_{n}$ & 0.972 & 0.8 \\
Tangential restitution $e_{t}$ & 0.25 & 0.35 \\
Friction coefficient $\mu$ & 0.33 & 0.596 \\
Spring stiffness $k_{n}(m g / D)$ & $2 \times 10^{5}$ & $2 \times 10^{5}$ \\
\hline
\end{tabular}

The equations of motion -including rotation - of each particle are calculated by applying Newton's second law and a Velocity-Verlet scheme is used for the numerical integration with a time step $d t=10^{-4}(\sqrt{D / g})$. We repeat the whole approach for grain-wall interactions. The simulation consists of confined granular flows between side walls on flat inclined base in the gravity field. The periodic 
boundary conditions (PBC) are used in the $X$-direction (figure 1).

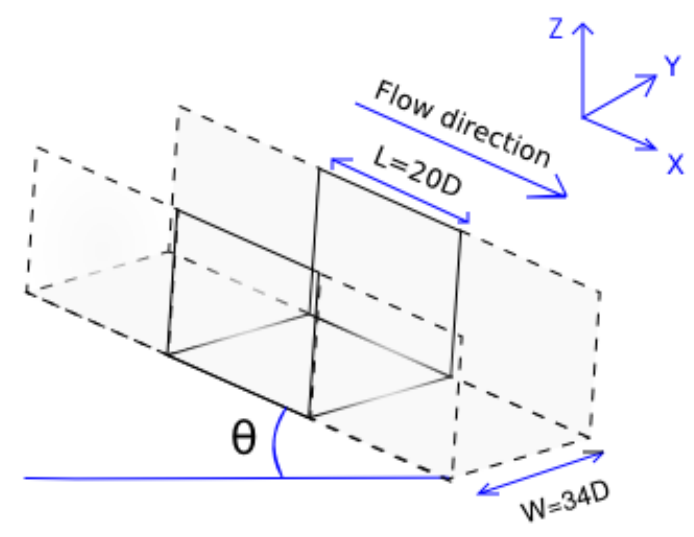

Figure 1: Sketch of the simulation configuration. The granular flow is confined by lateral walls with a gap $W$. Periodic boudary conditions are applied in the flow direction $x$ with a period $L$. The bottom has an angle of inclination $\theta$ with the horizontal.

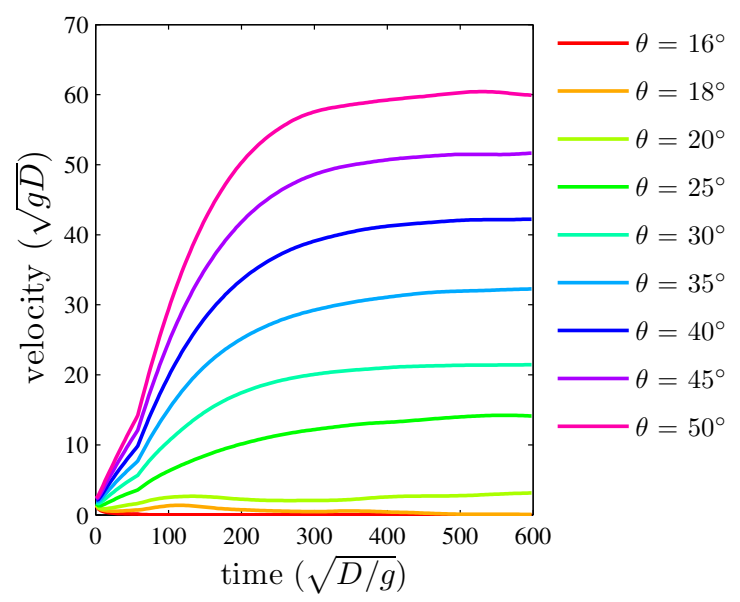

Figure 2: Temporal evolution of the averaged velocity for $W=34 D, \tilde{H}=8 D$. The steady-state is reached after an exponential saturation.

PBC are generally used to study steady and fully developed flows [6]. The periodic simulation cell has a length $L=20 D$ and a width $W=34 D$. In these numerical simulations, the control parameters are the angle of inclination $\theta$ and the mass hold up $\tilde{H}$. The mass hold up is an equivalent grain height corresponding to the mass per unit basal surface within the simulation box: $\tilde{H}=\frac{1}{\rho D} \sum_{g} \frac{m_{g}}{A}$ where $\rho$ denotes the grain density, $m_{g}$ is the mass of grain $g$ and $A=L \times W$ is the basal area of the box. We run simulations for $\tilde{H}=4,6,8 D$ varying inclination angle between 16 and $50^{\circ}$. For each value of the control parameters, the simulations are run up to a stabilization of the kinetic energy of the set of flowing grains, which is always achieved after about 300 time units. Simulations lead to SFD flows.
The flow shows a transient regime described by an exponential saturation (Figure 2) of the average grain velocity. The saturated velocity $V_{L}$ is an increasing function of the mass holdup and of the inclination angle.

\section{Transition to the supported regime}

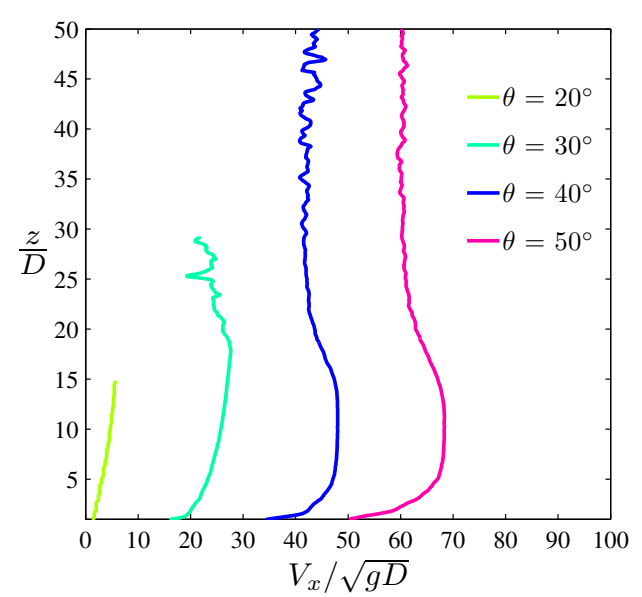

(a)

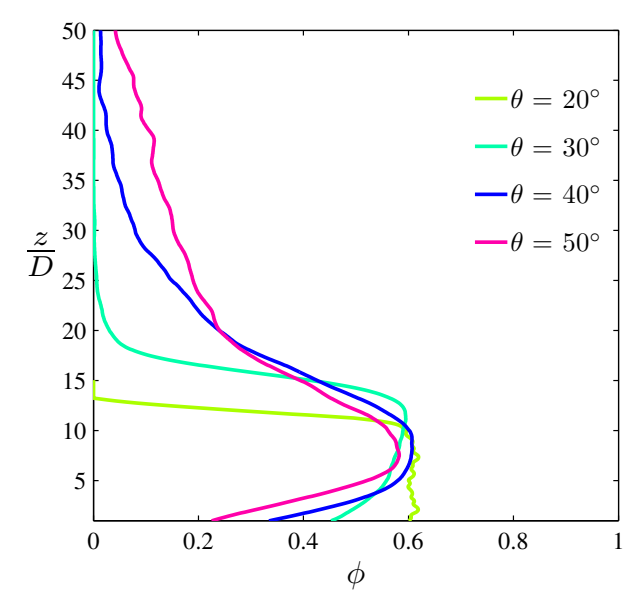

(b)

Figure 3: Height profiles of (a) the velocity in the main flow direction $\mathrm{X}$ and (b) the volume fraction, for $W=34 D$, $\tilde{H}=8 D$ and different angles of inclination $\theta$. These quantities are measured at the center of the channel and averaged over $4 D$ in the transverse direction.

Figure 3 shows the velocity and the volume fraction profiles for SFD flows at $\tilde{H}=8 D$ for $\theta=20^{\circ}, \theta=30^{\circ}$, $\theta=40^{\circ}$ and $50^{\circ}$. To get these profiles, data are averaged over the $\mathrm{X}$-direction and over 300 time units and then averaged along Y-direction over $+/-2 \mathrm{D}$ from the channel center. At $\theta=20^{\circ}$ the SFD flow is unidirectional with a volume fraction which does not vary much in $\mathrm{Z}(\phi \approx 0.6)$ and with layering (figure $4 a)$. The velocity profile is similar to what was obtained in [5] in the unidirectional regime: Bagnold profile with a slip velocity at the base. At $\theta=30^{\circ}$ 


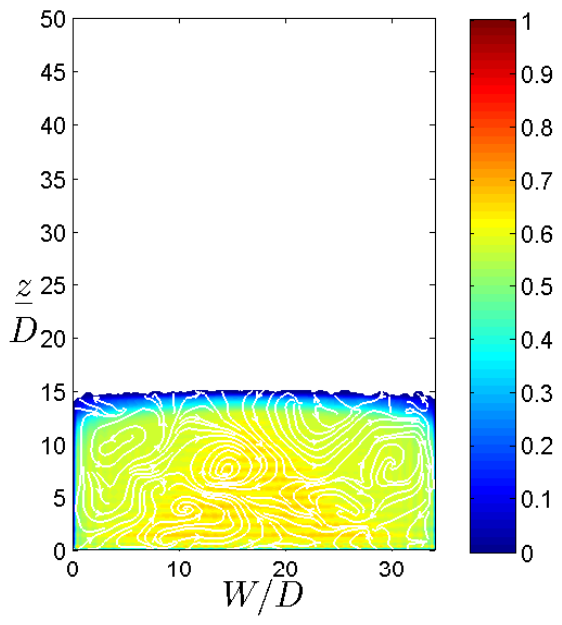

(a)

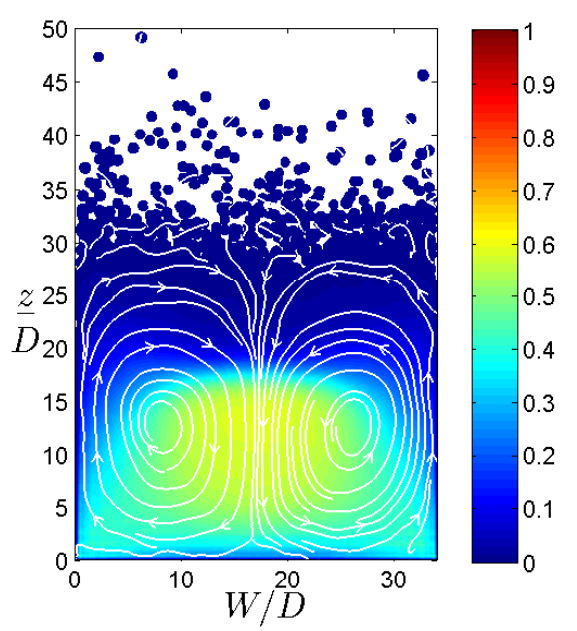

(b)

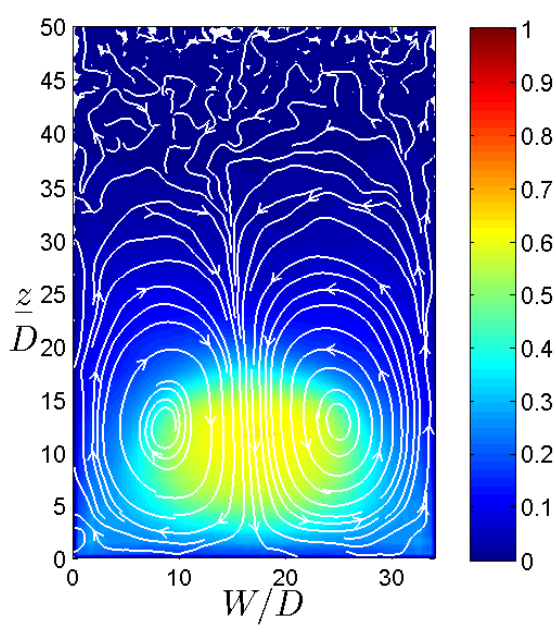

(c)

Figure 4: Two-dimensional maps representing the particle volume fraction and the velocity in the transverse direction, with the streamlines in white color, for $W=34 D$, $\tilde{H}=8 D$, (a) $\theta=20^{\circ}$, the particle volume fraction is higher at the base, (b) $\theta=30^{\circ}$, the streamline shows the presence of a convective regime (c) $\theta=35^{\circ}$, the dense core is clearly separated from the sidewalls and the base.

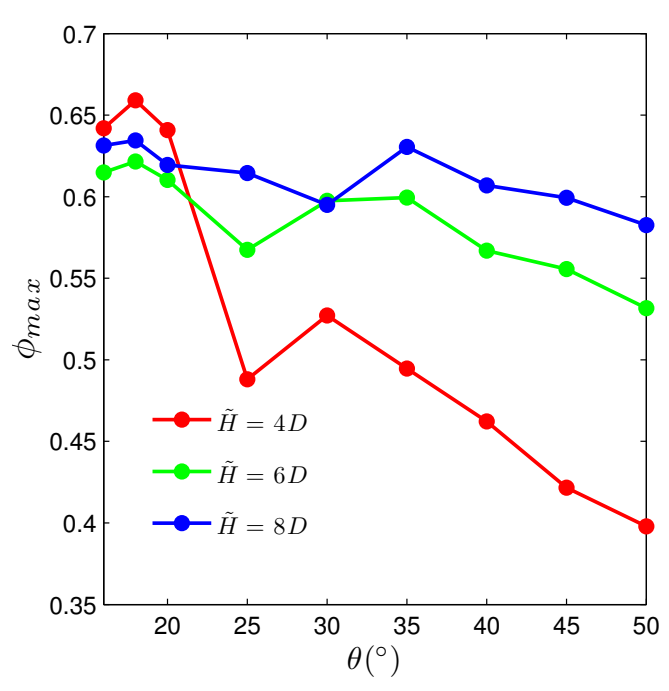

Figure 5: Maximum volume fraction $\phi_{\max }$ on the height profile as a function of $\theta$. The maximum volume fraction first decreases when the angle of inclination increases, then $\phi_{\max }$ increases indicating the transition to supported regime.

the unidirectional flow is destabilized and exhibits longitudinal rolls. Figure $3 \mathrm{~b}$ shows a lower volume fraction at the base than in the center of the flow. However, this depletion remains moderate and located at the flow base. The whole width of the channel is occupied by a pair of longitudinal vertices as illustrated by the streamlines in figure $4 \mathrm{~b}$. We find at higher angles of inclination $\left(\theta>30^{\circ}\right)$ a dense core with a fast and quasi-uniform longitudinal velocity above a basal layer moving at a slower speed. The presence of the dense core is illustrated in Figure $3 \mathrm{~b}$ by density profiles strongly inverted for $\theta>30^{\circ}$. The dense core floats above a very diluted and agitated granular layer. Furthermore, the dense core is also separated from sidewalls by strongly depleted regions (figure $4 \mathrm{c}$ ). We tried to find a simple criterion to quantify the transition toward supported regime. The variation of the maximum value of the volume fraction $\phi_{\max }$ within the flow with the inclination seems to give a good criterion, at least for small values of $\tilde{H}$. Figure 5 shows the variation of $\phi_{\max }$ for $\tilde{H}=4,6,8 D$ as a function of the angle of inclination $\theta$. At low inclination angles, $\phi_{\max }$ decreases when $\theta$ increases. This decrease is interrupted by a sudden increase of $\phi_{\max }$ corresponding to the transition toward supported regime. Then, in the supported regime, $\phi_{\max }$ diminishes again with increasing $\theta$ but at a lower rate.

We report, in Figure 6 the domains of existence of the rolls and supported regimes for $W=34 D$ and $68 D$ [1]. We indicate also the transition zone between the two regimes for both values of $W$. In [1], for $W=68 D$, the transition from rolls to the supported regime is identified between $\theta=25^{\circ}$ and $30^{\circ}$ for $4 D<\tilde{H}<8 D$. In our study, at $W=34 D$, this transition occurs between $\theta=25^{\circ}$ and $30^{\circ}$ for $\tilde{H}=4,6 D$. The same transition occurs between $\theta=30^{\circ}$ and $35^{\circ}$ for $\tilde{H}=8 D$. We thus notice increase of the transition angle at $\tilde{H}=8 D$. This increase of the 
transition angle can be also clearly observed for $W=68 \mathrm{D}$, in the phase diagram established by [1], but at higher mass hold up $(\tilde{H}>10 D)$.

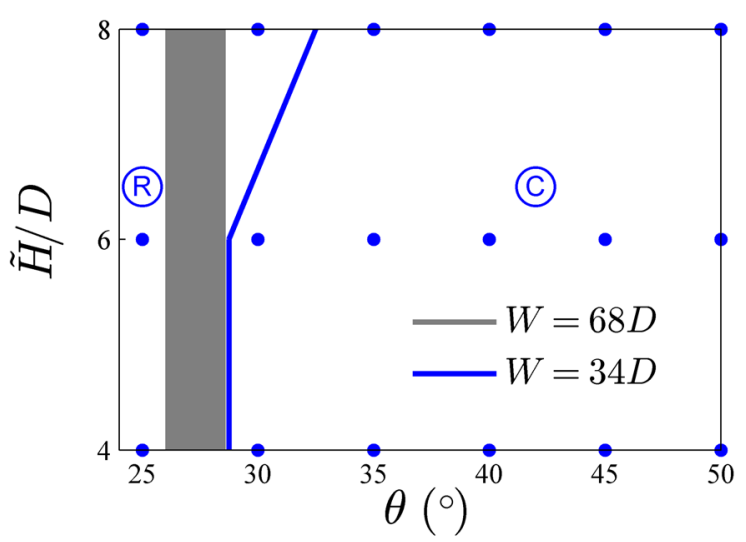

Figure 6: Phase diagram in mass hold up $(\tilde{H}=4$ to $8 D)$ angle of inclination $\left(\theta=25^{\circ}\right.$ to $\left.50^{\circ}\right)$ space. Blue dots are the sampling points where we performed simulations with a cell width $W=34 D$. Regimes: $\mathrm{R}$, convective flows with rolls [5, 7, 8], and (C), supported flows [1]. Grey band and blue line are the transition region from convective toward supported regime for $W=68 D$ and $34 D$ respectively.

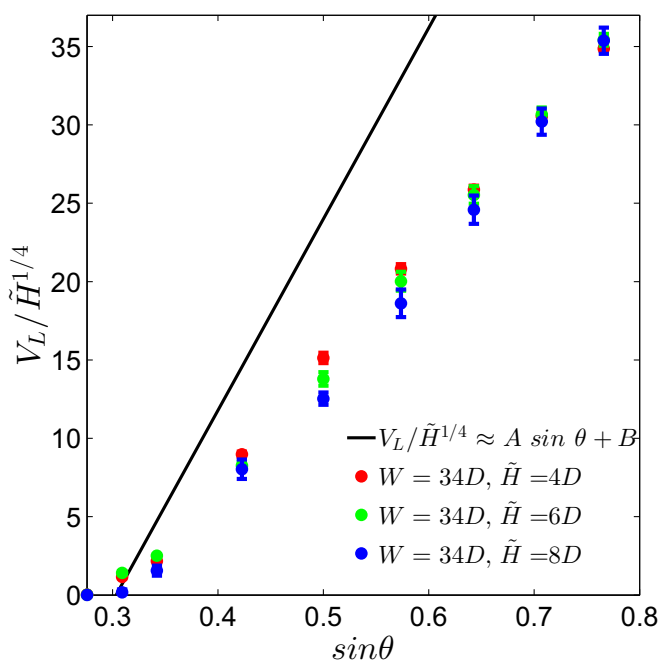

Figure 7: Rescaled limit velocity $V_{L} / \tilde{H}^{1 / 4}$ as a function of $\sin \theta$, for $\tilde{H}=4,6,8 D$ respectively, with $W=34 D$. The black curve represents the scaling law established by [1] for $W=68 D$. Error bars represent one standard deviation of the fluctuations of $V_{L}$

\section{Scaling law}

As highlighted in [1], for $W=68 D$, the mass flow rate obeys a scaling law giving the dependence of the limit velocity $V_{L}$ (figure 2 ) on the mass hold up and the inclination angle: $V_{L} / H^{1 / 4} \approx A \sin \theta+B$ with $A \approx 122$ and $B \approx-37$. We thus calculated, for $W=34 D$, the limit velocity $V_{L}$ - by averaging in time over 300 time units in the stationary regime the average grain velocity - rescaled by $\tilde{H}^{1 / 4}$, in function of the inclination angle $\theta$ for various mass hold up (figure 4). Our results follow the same scaling law but with a different slope. We can thus suspect that the scaling law of $V_{L}$ in $\tilde{H}^{1 / 4}$ is universal, with coefficients $A$ and $B$ varying with $W$ in a way which remains to be specified. This should be the object of further studies.

\section{Conclusion}

We performed numerical simulations of confined granular flow with a cell width $W=34 D$. Even for this relatively small value we observed the longitudinal rolls reported in $[1,7,8]$. We found that SFD supported regime appears for angles of inclination $\theta>30^{\circ}$. This is consistent with the results obtained with $W=68 D$ [1]. The transition toward the supported regime seems to be characterized by a sudden increase of the maximum volume fraction with the angle of inclination after a monotonous decrease. We recover, for $W=34 D$, the mass flow rate scaling law highlighted in [1]. This encourages systematic investigations for other values of $W$.

\section{References}

[1] N. Brodu, R. Delannay, A. Valance, P. Richard, Journal of Fluid Mechanics 769, 218-228 (2015).

[2] P. Jop, Comptes Rendus Physique 16, 62-72 (2015).

[3] E. Martínez, A. González-Lezcano, A. J. BatistaLeyva, E. Altshuler, Physical Review E 93, 062906 (2016).

[4] G. G. Zhou, C. W. Ng, Q. C. Sun, Landslides, 11, 369384 (2014).

[5] N. Brodu, P. Richard, R. Delannay, Physical Review E 87(2), 022202 (2013).

[6] N. Taberlet, Ecoulements gravitaires de matériaux granulaires (PhD thesis, Université Rennes 1, 2005).

[7] Y. Forterre, O. Pouliquen, Journal of Fluid Mechanics, 467, 361-387 (2002).

[8] T. Börzsönyi,R. E. Ecke, J.N. McElwaine, Physical review letters, 103, 178302 (2009). 